\title{
Магнитоспектроскопия двойных квантовых ям HgTe/CdHgTe
}

\author{
(C) Л.С. Бовкун ${ }^{+}$, С.С. Криштопенко ${ }^{+\ddagger}$, А.В. Иконников ${ }^{+\dagger}$, В.Я. Алешкин ${ }^{+\dagger}$, А.М. Кадыков ${ }^{+\ddagger}$, \\ S. Ruffenach ${ }^{\ddagger}$, C. Consejo ${ }^{\ddagger}$, F. Teppe ${ }^{\ddagger}, W$. Knap $\ddagger$, M. Orlita ${ }^{\S}$, B. Piot ${ }^{\S}$, M. Potemski ${ }^{\S}$, \\ Н.Н. Михайлов ${ }^{*} \dagger$, С.А. Дворецкий* , В.И. Гавриленко ${ }^{+\dagger}$ \\ + Институт фризики микроструктур Российской академии наук, \\ 603950 Нижний Новгород, Россия \\ ${ }^{\ddagger}$ Laboratoire Charles Coulomb (L2C), UMR CNRS 5221 \& UM, \\ 34095 Montpellier, France \\ † Нижегородский государственный университет им. Н.И. Лобачевского, \\ 603950 Нижний Новгород, Россия \\ $\S$ Laboratoire National des Champs Magnetiques Intenses (LNCMI-G), CNRS-UJF-UPS-INSA, \\ FR-38042 Grenoble, France \\ * Институт ффизики полупроводников им. А.В. Ржанова Сибирского отделения Российской академии наук, \\ 630090 Новосибирск, Россия \\ ${ }^{\dagger}$ Новосибирский государственный университет, \\ 630090 Новосибирск, Россия \\ E-mail: antikon@ipmras.ru
}

(Получена 18 мая 2016 г. Принята к печати 23 мая 2016 г.)

Исследованы спектры магнитопоглощения в двойных квантовых ямах $\mathrm{HgTe} / \mathrm{CdHgTe}$ с нормальным и инвертированным зонным спектром. На основе модели Кейна 8.8 рассчитаны уровни Ландау в симметричных квантовых ямах с прямоугольным профилем потенциала. Показано, что наличие туннельнопрозрачного барьера приводит к расщеплению состояний и „удвоению“ основных линий магнитопоглощения. При ширине ям, близкой к критической, для структуры с одиночной квантовой ямой показаны наличие инверсии зон и возникновение бесщелевой зонной структуры, как в двухслойном графене. Обнаружен сдвиг линий магнитопоглощения при изменении концентрации носителей за счет эффекта остаточной фотопроводимости, связываемый с изменением профиля потенциала при перезарядке ловушек, что открывает возможность управления топологическими фазовыми переходами в таких структурах.

\section{1. Введение}

Гетероструктуры с квантовыми ямами (КЯ) на основе $\mathrm{HgTe} / \mathrm{CdHgTe} \mathrm{в} \mathrm{последние} \mathrm{годы} \mathrm{привлекают} \mathrm{большое}$ внимание исследователей как в России, так и в мире. В значительной мере этот интерес связан с тем, что КЯ $\mathrm{HgTe} / \mathrm{CdHgTe} \mathrm{с} \mathrm{инвертированной} \mathrm{зонной} \mathrm{структу-}$ рой (двумерные топологические изоляторы) являются первой системой, в которой показано существование защищенных краевых каналов [1] (недавно схожие результаты стали получать и для структур InAs/GaSb). Исследования последних лет выявили у таких структур ряд других замечательных свойств: гигантское (до 30 мэВ) спиновое расщепление Рашбы [2,3], полуметаллическое состояние вследствие перекрытия валентной зоны и зоны проводимости в широких КЯ $[4,5]$, состояние трехмерного топологического изолятора в напряженных пленках HgTe [6]. Зонная структура КЯ $\mathrm{HgTe} / \mathrm{CdHgTe}$ перестраивается от нормальной (СdTе-подобной) до инвертированной при увеличении ширины КЯ $\mathrm{HgTe}$. При критической ширине КЯ $d_{c} \approx 6.3$ нм зона проводимости и валентная зона смыкаются, а закон дисперсии носителей заряда становится линейным (безмассовые дираковские фермионы), как в однослойном графене [7]. Соответственно можно ожидать, что зонная структура двойной КЯ HgТе при определенных условиях будет аналогична зонной структуре двухслойного графена [8].
Однако наличие туннельно-прозрачного в двойной КЯ $\mathrm{HgTe}$ открывает дополнительные возможности для инженерии энергетического спектра по сравнению с двухслойным графеном. До недавнего времени зонная структура двойных КЯ HgТе исследовалась лишь в работах группы P. Michetti [9-11] на основе обобщения „двухзонной“ м модели BHZ (Bernevig-Hughes-Zhang) [8]. Следует отметить, что модель $\mathrm{BHZ}$ даже в одиночной КЯ HgТе применима в весьма ограниченной области энергий [7], а область применимости „обобщенной модели Michetti“ к двойным КЯ до настоящего момента не исследовалась. Первое теоретическое исследование зонной структуры двойных КЯ $\mathrm{HgTe} / \mathrm{CdHgTe}$ на основе 8-зонной модели Кейна выполнено в работах [12,13], где была построена фазовая диаграмма симметричных двойных КЯ $\mathrm{HgTe} / \mathrm{CdHgTe} \mathrm{в} \mathrm{зависимости} \mathrm{от} \mathrm{толщин} \mathrm{сло-}$ ев ямы $\mathrm{HgTe}$ и разделительного туннельно-прозрачного барьера $\mathrm{CdHgTe}$ В частности, было обнаружено, что при инверсии зон система переходит в специфическую бесщелевую фазу, существующую в широком интервале значений толщин слоев, которая проявляет многие свойства, присущие двухслойному графену. В настоящей работе выполнены первые экспериментальные исследования зонной структуры двойных КЯ НgТе с нормальным и инвертированным зонным спектром методами магнитоспектроскопии в дальнем и среднем инфракрасных диапазонах. Результаты интерпретированы на основе 
выполненных в рамках модели Кейна $8 \cdot 8$ расчетов уровней Ландау.

\section{2. Методы расчета}

Зонная структура двойных КЯ $\mathrm{HgTe} / \mathrm{CdHgTe}$ рассчитывалась с использованием 8-зонного $\mathbf{k} \cdot \mathbf{p}$-гамильтониана в качестве оператора кинетической энергии. В используемом гамильтониане $\mathbf{k} \cdot \mathbf{p}$-взаимодействие зоны проводимости $\Gamma_{6}$ с валентными зонами $\Gamma_{8}$ и $\Gamma_{7}$ рассматривается точно, а взаимодействие с остальными зонами учитывается по теории возмущений. Такой подход позволяет корректным образом учитывать непараболичность подзон размерного квантования, спин-орбитальное взаимодействие и величину встроенных упругих деформаций, обусловленных различием постоянных решеток материалов в плоскости КЯ, для структур, выращенных на буфере $\mathrm{CdTe}$. Расчет волновых функций был выполнен методом, описанным в работе [14]. Суть метода расчета состоит в рассмотрении вместо одной двойной КЯ сверхрешетки с достаточно большим периодом $d$. Из-за большой толщины барьеров между соседними двойными КЯ в сверхрешетке туннелированием носителей заряда можно пренебречь и в качестве граничных условий использовать условие периодичности волновой функции с периодом $d$. Электронная волновая функция в этой модели имеет 8 компонент. Каждая компонента разлагалась по пространственным гармоникам:

$$
\begin{aligned}
\psi_{j}(x, y, z) & =\exp \left(i k_{x} x+i k_{y} y\right) \sum_{n=-N_{\max }}^{N_{\max }} C_{n}\left(k_{x}, k_{y}\right) \exp \left(i \frac{2 \pi z}{d} n\right) \\
& =\exp \left(i k_{x} x+i k_{y} y\right) \varphi_{j}\left(k_{x}, k_{y}, z\right)
\end{aligned}
$$

где $k_{x, y}$ - компоненты волнового вектора электрона в КЯ, z - координата, нормальная к плоскости КЯ, $2 N_{\max }+1$ - число пространственных гармоник в разложении, индекс $j$ обозначает компоненту волновой функции, $C_{n}\left(k_{x}, k_{y}\right)$ - коэффициенты разложения. Далее полагалось, что КЯ выращена на плоскости (013) и $x\|[100], y\|[03 \overline{1}], z \|[013]$. Нахождение спектра и волновых функций при таком подходе сводится к нахождению собственных значений и собственных векторов матрицы размерностью $8\left(2 N_{\max }+1\right) \times 8\left(2 N_{\max }+1\right)$. При расчетах использовались материальные параметры из работы [15].

\section{3. Эксперимент}

Исследуемые образцы были выращены методом молекулярно-лучевой эпитаксии (МЛЭ) с эллипсометрическим контролем состава и толщины слоев на полуизолирующих подложках $\mathrm{GaAs}$ (013) [16,17]. Активная часть структуры выращивалась на толстом релаксированном буферном слое $\mathrm{CdTe}$ и состояла из нижнего барьерного слоя $\mathrm{Hg}_{1-x} \mathrm{Cd}_{x}$ Те толщиной 30 нм, двух КЯ $\mathrm{HgTe}$ шириной $d_{\mathrm{QW}}$, разделенных туннельно-прозрачным
Параметры исследованных образцов

\begin{tabular}{c|c|c|c}
\hline Номер образца & $x_{\text {bar }}$ & $d_{\mathrm{QW}}, \mathrm{HM}$ & $t_{\mathrm{bar}}, \mathrm{HM}$ \\
\hline $1(150217)$ & 0.64 & $4.5(3.8)$ & $3(3.7)$ \\
$2(150218)$ & 0.71 & $6.6(6.5)$ & 3
\end{tabular}

барьером $\mathrm{Hg}_{1-x} \mathrm{Cd}_{x}$ Те толщиной $t_{\mathrm{bar}}$ и верхнего барьера $\mathrm{Hg}_{1-x} \mathrm{Cd}_{x}$ Te толщиной 30 нм. Поверх выращивался покрывающий слой CdTe толщиной 40 нм. Структуры специально не легировались. Ростовые параметры структур приведены в таблице. Они выбирались таким образом, чтобы образец 1 (150217) имел нормальную зонную структуру, а образец 2 (150218) соответствовал фазе „Двухслойного графена“. В скобках указаны „уточненные“ параметры, полученные из условия наилучшего согласия наблюдаемых и рассчитанных энергий магнитооптических переходов.

Измерения спектров магнитопоглощения проводились в Национальной лаборатории сильных магнитных полей в Гренобле (Франция) и в Лаборатории им. Шарля Кулона Национального центра научных исследований и Университета Монпелье (Франция) методом фурьеспектроскопии с разрешением 4-8 $\mathrm{cm}^{-1}$ при температуpe $T=4.2 \mathrm{~K}$ в магнитных полях до 15 Тл в геометрии Фарадея на образцах размером $5 \times 5$ мм. В качестве источника излучения использовался глобар или ртутная лампа, в качестве приемника - композиционный кремниевый болометр. Измеренные спектры пропускания нормировались на спектр пропускания в нулевом магнитном поле. Концентрация носителей заряда могла изменяться при кратковременной (за счет эффекта остаточной фотопроводимости) или непрерывной подсветке образца излучением синего светодиода, расположенного в жидком гелии рядом с образцом. Для определения концентрации методом Ван-дер-Пау по углам образца располагались четыре точечных индиевых контакта.

\section{4. Результаты и обсуждение}

На вставке к рис. 1 представлены рассчитанные зависимости от волнового вектора $k$ энергий $E$ двух нижних электронных подзон $E_{1}, E_{2}$ и двух верхних дырочных подзон $H_{1}, H_{2}$ в образце 150217 с нормальной зонной структурой. Видно, что для электронных состояний барьер является туннельно-прозрачным (подзоны $E_{1}$ и $E_{2}$ расщеплены), в то время как подзоны $H H_{1}$ и $\mathrm{HH}_{2}$ вырождены при $k=0$. Рассчитанные в аксиальном приближении уровни Ландау для этого образца представлены на рис. 1. Видно, что расщепление подзон в зоне проводимости приводит к возникновению двух идентичных вееров уровней Ландау. В валентной зоне, где при $k=0$ расщепление отсутствовало, приложение магнитного поля приводит к небольшим расщеплениям уровней Ландау дырок кроме уровня $n=-2$, который остается „двукратно“ вырожденным (по числу КЯ). 


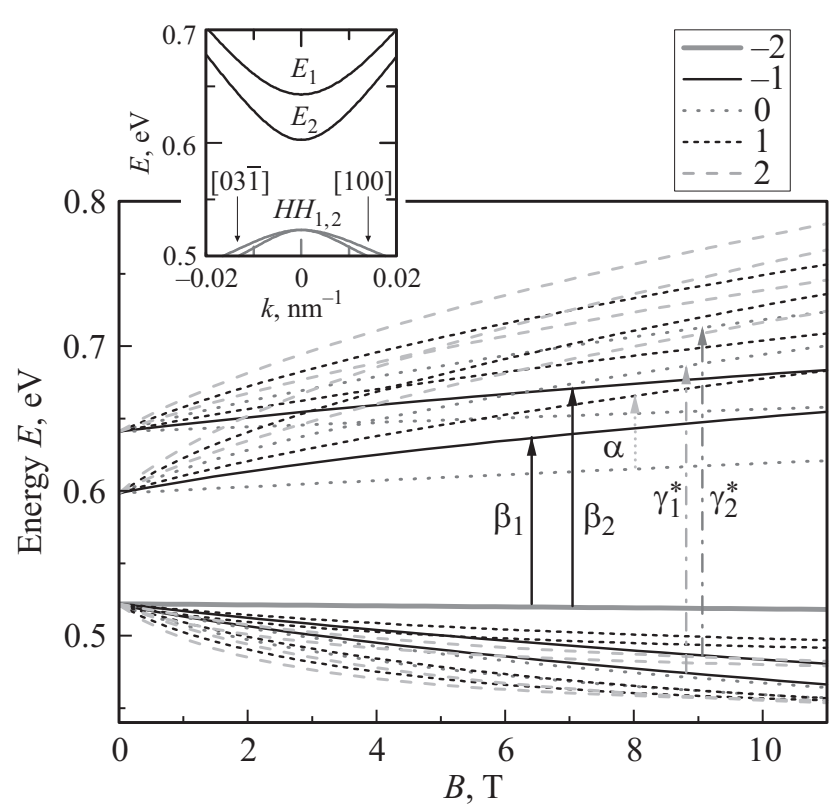

Рис. 1. Рассчитанные уровни Ландау в гетероструктуре 150217 с двойной квантовой ямой НgТе. Стрелками показаны наблюдаемые магнитооптические переходы. На вставке - рассчитанная зонная структура образца в отсутствие магнитного поля.

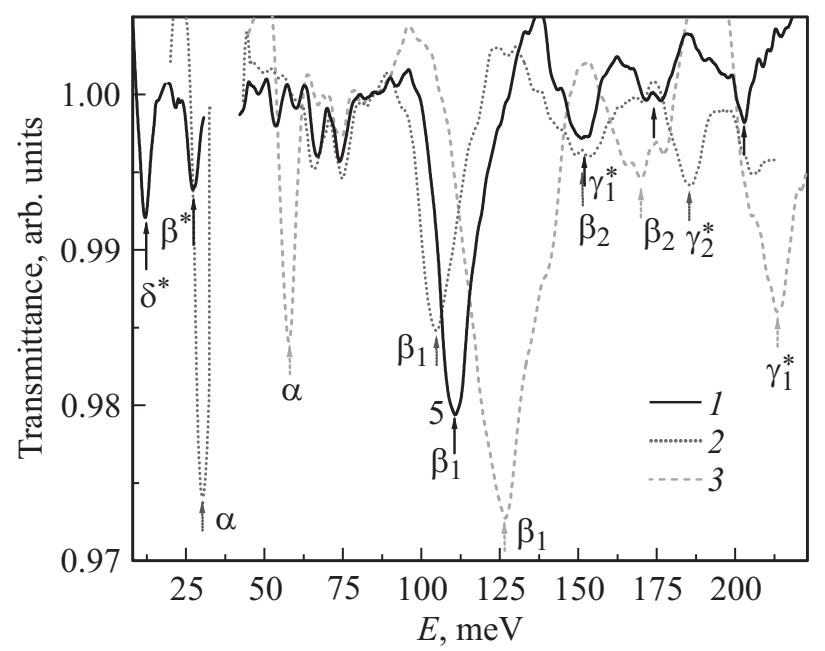

Рис. 2. Спектры магнитопоглощения в гетероструктуре 150217 , измеренные в темновых условиях при $B=5$ Тл $(1)$ и в условиях подсветки синим светодиодом при $B=3.75$ (2) и 8.75 Тл (3).

На рис. 2 представлены типичные спектры магнитопоглощения, а на рис. 3 - положения линий, наблюдаемых при разных уровнях подсветки, в зависимости от магнитного поля в образце 150217. Как показали измерения магнитотранспорта, в темновых условиях образец имеет дырочный тип проводимости с концентрацией дырок $p \approx 5 \cdot 10^{10} \mathrm{~cm}^{-2}$. При длительной подсветке синим светодиодом тип проводимости меняется на электронный с концентрацией электронов $n \approx 5 \cdot 10^{10} \mathrm{~cm}^{-2}$. Поэтому в темновых условиях в этом образце могут наблюдаться переходы между уровнями Ландау дырок и межзонные переходы, а при подсветке переходы в валентной зоне должны сменяться циклотронным резонансом электронов. Как видно из рис. 2,3, в темновых условиях в образце действительно наблюдаются две низкочастотные линии поглощения $\delta^{*}$ и $\beta^{*}$, которые „выключаются“ при подсветке образца. Подобные линии магнитопоглощения дырок в квантующих магнитных полях наблюдались ранее в напряженных квантовых ямах Ge и InGaAs, где верхняя подзона валентной зоны образована, как и в данном случае, состояниями тяжелых дырок $[18,19]$. Эти две линии связывались с переходами с участием двух верхних уровней Ландау: $-1 \rightarrow-2\left(\beta^{*}\right)$ и $2 \rightarrow 1\left(\delta^{*}\right)$. Однако, как видно из рис. 3, наблюдаемые положения линий $\delta^{*}$ и $\beta^{*}$ не вполне согласуются с рассчитанными в рамках аксиальной модели расстояниями между соответствующими уровнями Ландау дырок. Такое несоответствие отмечалось и для одиночных КЯ $\mathrm{HgTe} / \mathrm{CdHgTe} \mathrm{[20].} \mathrm{По} \mathrm{нашему} \mathrm{мнению,} \mathrm{это} \mathrm{обусловле-}$ но сильной гибридизацией уровней Ландау вследствие замешивания состояний легких и тяжелых дырок на гетероинтерфейсе [21], приводящего к расщеплению состояний и сильному возмущению закона дисперсии в ограниченной области $k$-пространства. Соответственно (и это показывают наши предварительные расчеты) это должно приводить к перестройке спектра близко расположенных уровней Ландау дырок в некоторой области магнитных полей. Поэтому в дальнейшем при интерпретации наблюдаемых линий магнитопоглощения мы будем исходить из их поведения в сильных магнитных полях, где влияние эффектов, связанных с замешиванием состояний на гетероинтерфейсах, должно уменьшаться.

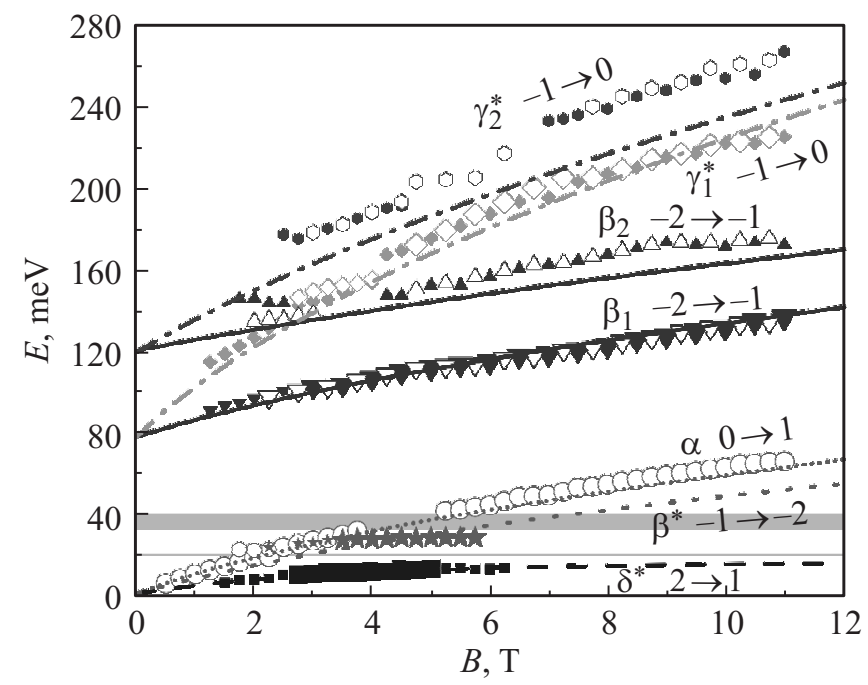

Рис. 3. Рассчитанные (линии) и измеренные (точки) положения линий магнитопоглощения в зависимости от магнитного поля в гетероструктуре 150217. Сплошные символы соответствуют темновым условиях, открытые символы - подсветке синим светодиодом; размеры символов указывают на интенсивность линии поглощения. 
Как уже отмечалось, при подсветке тип проводимости образца меняется на электронный, и в спектрах возникает линия поглощения $\alpha$. Поскольку в этом случае начиная уже с полей $B \sim 1$ Тл фактор заполнения уровней Ландау $<1$, эта линия, очевидно, связана с переходом с нижнего уровня Ландау зоны проводимости $0 \rightarrow 1$. В меньших магнитных полях, когда начинает заполняться следующий уровень Ландау, должен наблюдаться более низкочастотный переход $-1 \rightarrow 0$, однако в этой области частот падает чувствительность установки и переход не удается выделить на фоне шумов. Как видно из рис. 2, рассчитанная для „уточненных“ параметров структуры зависимость энергии этого перехода от магнитного поля в целом согласуется с данными эксперимента, хотя и очевидно систематическое превышение наблюдаемых энергий перехода над расчетными. Отметим, что наблюдаемая ширина линий $\delta^{*}, \beta^{*}$ и $\alpha$, связанных с переходами между уровнями Ландау, относящихся к одной подзоне (рис. 2), в несколько раз меньше, чем характерная ширина обсуждаемых далее линий межзонных переходов, что, скорее всего, связано с флуктуациями ширины КЯ (по площади структуры), приводящими к размытию ширины запрещенной зоны.

Обратимся теперь к анализу спектров магнитопоглощения в области высоких частот. Наиболее интенсивную линию поглощения $\beta_{1}$, которая наблюдается как в темновых условиях, так и при подсветке, естественно связать с межзонным переходом $-2 \rightarrow-1$ с верхнего уровня Ландау валентной зоны на уровень $n=-1$, относящийся к подзоне $E_{1}$. Этот переход должен наблюдаться и в темновых условиях (в образце проводимости $p$-типа) начиная с полей, соответствующих фактору заполнения уровней Ландау дырок $v=2$ (вследствие ,двукратного“ вырождения уровня $n=-2$ ), т. е. с полей $B \sim 1$ Тл, что и соответствует наблюдаемой картине (рис. 3). Линейная экстраполяция положения этой линии к нулевому магнитному полю дает значение ширины запрещенной зоны $\sim 80$ мэВ, которое использовалось в качестве одной из заданных величин при уточнении параметров структуры, закладываемых в расчет зонных спектров. Второй такой величиной было расщепление подзон $E_{1}$ и $E_{2} \sim 40$ мэВ, которое определялось из линейной экстраполяции к нулевому магнитному полю вышележащей линии $\beta_{2}$, которую мы связываем с переходом с уровня $n=-2$ на уровень $n=-1$, относящийся к подзоне $E_{2}$. Проведенные расчеты вероятностей межзонных переходов показывают, что интенсивности линий поглощения $\beta_{1}$ и $\beta_{2}$ должны кратно превосходить интенсивности других линий межзонных переходов. При этом интенсивность линии $\beta_{1}$ должна быть в 1.5-2 раза больше, чем интенсивность линии $\beta_{2}$. Однако, как видно из спектров, представленных на рис. 2, наблюдаемое соотношение интенсивностей заметно больше и составляет 3-4 раза. Причина такого несоответствия может заключаться в небольшом расщеплении уровней $n=-2$ вследствие различия в толщинах номинально одинаковых квантовых ям, в результате чего населенность верхнего из них оказывается меньше, что приводит к уменьшению интенсивности линии поглощения $\beta_{2}$. Заметим, что близкие (к переходам $-2 \rightarrow-1$ ) значения энергии имеют также переходы $1 \rightarrow 0$ с верхней пары уровней Ландау $n=1$ в валентной зоне на нижнюю пару уровней Ландау $n=0$ в зоне проводимости. Из четырех таких возможных переходов разрешенными, как показывает численный расчет, являются только переходы с нижнего на нижний и с верхнего на верхний уровни Ландау, однако их вероятности оказываются почти на порядок меньше, чем для переходов $-2 \rightarrow-1$.

При еще бо́льших энергиях квантов в спектрах видны достаточно слабые межзонные переходы, обозначенные как $\gamma_{1}^{*}$ и $\gamma_{2}^{*}$ (см. рис. 2,3). Исходя из наблюдаемых зависимостей энергий резонансов от магнитного поля и рассчитанных вероятностей переходов (вероятности межзонных переходов падают с увеличением номеров „вовлеченных“ уровней Ландау) мы связываем эти линии с переходами с пары уровней Ландау $n=-1$ в валентной зоне на вторую пару уровней Ландау $n=0$ в зоне проводимости (разрешенными опять оказываются переходы с нижнего на нижний и с верхнего на верхний).

Из рис. 3 видно, что, как и для линии $\alpha$, для межзонных переходов $\left(\beta_{2}, \gamma_{1}^{*}\right.$ и $\left.\gamma_{2}^{*}\right)$ расчет дает несколько заниженные значения наклона зависимости положения линии от магнитного поля. Это расхождение не удается полностью устранить варьированием в разумных пределах структурных параметров исследуемого образца (по сравнению с ростовыми данными). Аналогичные расхождения фиксировались ранее и для одиночных КЯ HgTe/CdHgTe [22,23]. Скорее всего, эти расхождения обусловлены как несовершенством используемой аксиальной модели закона дисперсии (не учитывающей эффектов понижения симметрии, связанных с отсутствием центра инверсии кристаллической решетки и замешиванием дырочных состояний на гетероинтерфейсах), так и неточностью определения используемых в расчетах материальных параметров гетеропары $\mathrm{HgTe} / \mathrm{CdTe}$. Для решения этих проблем требуются дополнительные исследования на простых модельных структурах, к которым никак нельзя отнести двойные КЯ. Главным результатом исследования двойной КЯ с нормальной зонной структурой (образец 150217) является наблюдение удвоения линий межзонных переходов, свидетельствующего о реализации туннельно-прозрачного барьера и расщепления состояний в зоне проводимости.

Перейдем теперь к обсуждению результатов исследования второй гетероструктуры — 150218. В этом образце толщина каждой из квантовых ям близка к критической (для структуры с одиночной КЯ), но вследствие туннельно-прозрачного (для электронных состояний) разделительного барьера зонный спектр становится инвертированным. Как видно из вставки к рис. 4, в этом образце происходит инверсия подзон $E_{1}$ и $H H_{1}$. В результате реализуется бесщелевая зонная структура, образованная касающимися в точке $k=0$,параболическими“ подзонами $H H_{1}$ и $H H_{2}$, как в двухслойном 


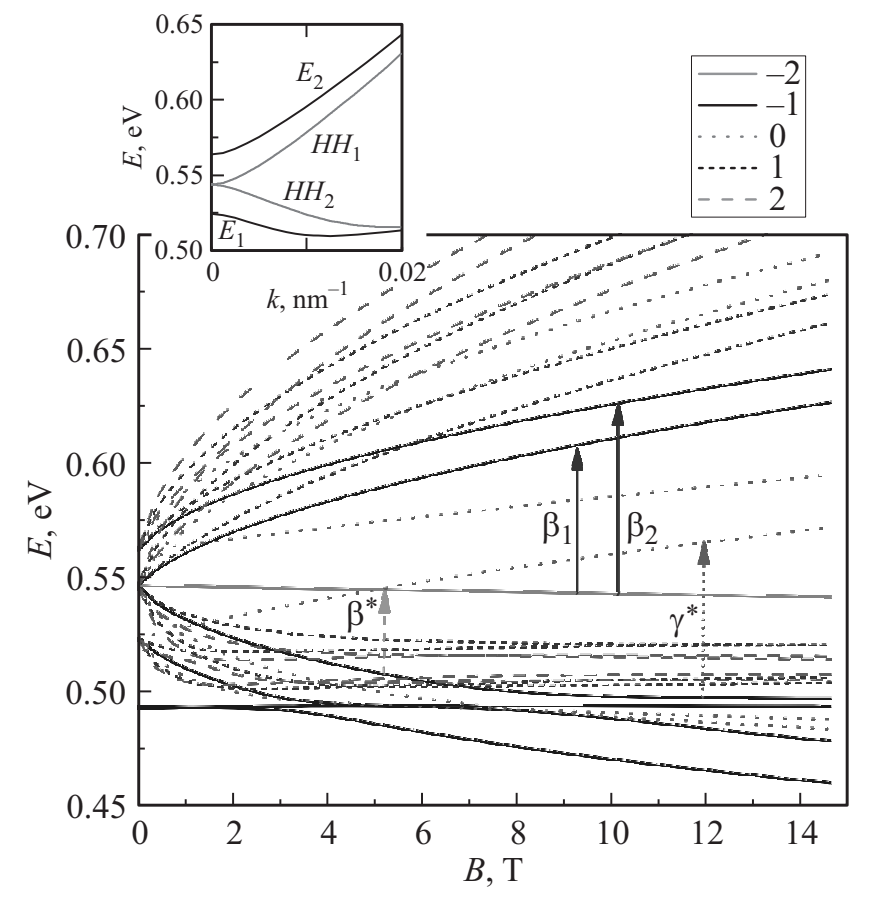

Рис. 4. Рассчитанные уровни Ландау в гетероструктуре 150218 с двойной квантовой ямой HgТе. Стрелками показаны наблюдаемые магнитооптические переходы. На вставке - рассчитанная зонная структура образца в отсутствие магнитного поля.

графене. Вырождение подзон $\mathrm{HH}_{1}$ и $\mathrm{HH}_{2}$ при $k=0$ связано с непрозрачностью разделительного барьера для дырочноподобных состояний. На рис. 4 представлены рассчитанные в аксиальном приближении уровни Ландау для этого образца. Видно, что, как и в образце 150217 , в этой двойной КЯ происходит удвоение числа уровней Ландау с одинаковыми номерами (уровень $n=-2$ остается двукратно вырожденным), однако инверсия зон $E_{1}$ и $H H_{1}$ проявляется в том, что нижний из уровней $n=0$, относящийся, как и в образце 150217 , и в сильных магнитных полях к зоне проводимости, пересекает с уменьшением поля при некотором его значении $B_{c} \approx 4.6$ Тл уровни $n=-2$ и становится принадлежащим, таким образом, валентной зоне (соответственно ниже $B_{c}$ один из уровней $n=-2$ относится к зоне проводимости).

На рис. 5 представлены типичные спектры магнитопоглощения, а на рис. 6 - положения линий, наблюдаемых при разных уровнях подсветки, в зависимости от магнитного поля в образце 150218. Этот образец при всех уровнях подсветки имеет $p$-тип проводимости, концентрация дырок падает от $p \approx 1.5 \cdot 10^{11}$ в темновых условиях до $\sim 2 \cdot 10^{10} \mathrm{~cm}^{-2}$ при длительной (или непрерывной) подсветке синим светодиодом. Поэтому в этом образце могут наблюдаться переходы между уровнями Ландау дырок, а также межзонные переходы. Как видно из рис. 5,6, в низкочастотной области (до 20 мэВ) в образце наблюдается линия поглощения $\mathrm{CR}_{h}$, положение которой существенно смещается в область больших энергий при подсветке (уменьшении концентрации дырок). При этом резонансная частота при всех концентрациях линейно зависит от магнитного поля. В темновых условиях линия $\mathrm{CR}_{h}$ наблюдается вплоть до магнитных полей $B \approx 2$ Тл, что соответствует фактору заполнения уровней Ландау дырок $v=3$, при подсветке (и уменьшении концентрации дырок) эта линия исчезает в меньших магнитных полях. Все это позволяет связать линию $\mathrm{CR}_{h}$ с классическим циклотронным резонансом дырок. Положения остальных наблюдаемых линий поглощения слабо зависят от концентрации дырок (при подсветке

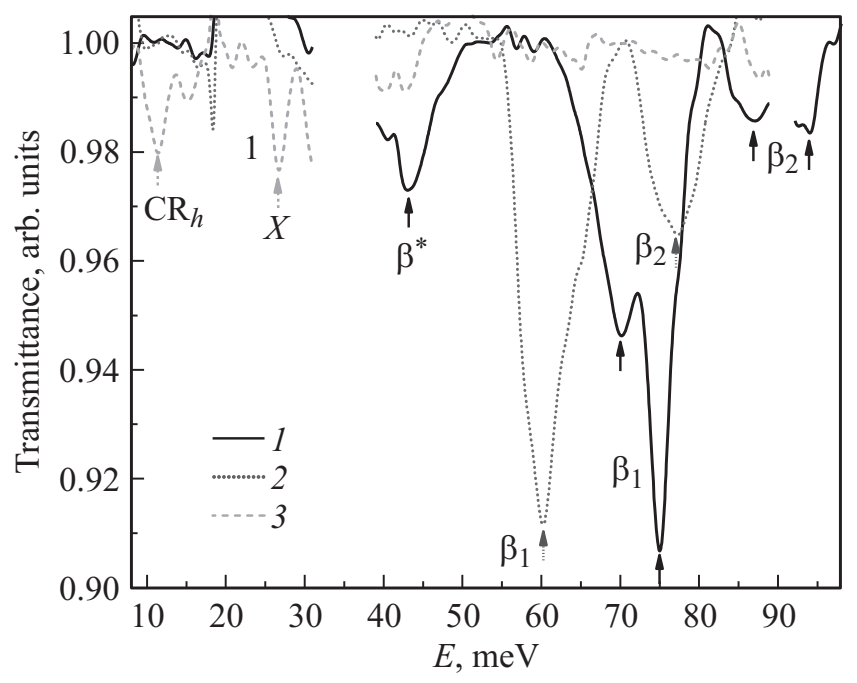

Рис. 5. Спектры магнитопоглощения в гетероструктуре 150218 , измеренные в темновых условиях при $B=5$ Тл (1) и в условиях подсветки синим светодиодом при $B=3.35$ (2) и 8.75 Тл $(3)$.

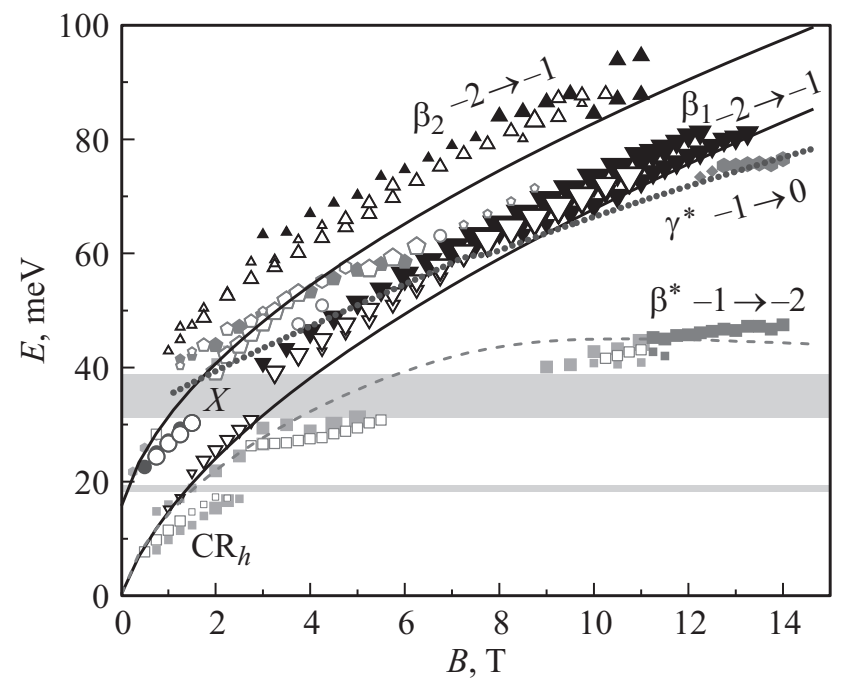

Рис. 6. Рассчитанные (линии) и измеренные (точки) положения линий магнитопоглощения в зависимости от магнитного поля в гетероструктуре 150218. Сплошные символы соответствуют темновым условиях, открытые символы - подсветке синим светодиодом. Размеры символов указывают на интенсивность линии поглощения. 
главным образом меняются их интенсивности), что указывает на их связь с переходами между вполне определенными уровнями Ландау. В настоящей работе мы ограничимся интерпретацией только наиболее интенсивных линий поглощения. Как для образца 150217 , мы будем в первую очередь исходить из поведения линий в сильных магнитных полях, где используемая теоретическая модель наиболее адекватна.

Начнем с линии $\beta^{*}$, интенсивность которой падает при подсветке, что указывает на ее связь с переходом (переходами) между уровнями Ландау валентной зоны. В темновых условиях $v=1$ при $B \approx 6$ Тл и в больших магнитных полях единственным не полностью заполненным электронами уровнем Ландау дырок является верхний уровень в валентной зоне $n=-2$, на который возможны переходы только с уровней $n=-1$. Как видно из рис. 4 , в сильных полях, $B>9$ Тл, в валентной зоне как раз имеется такой „подходящий“ уровень $n=-1$, происходящий из той же дырочной подзоны $H_{2}$, что и уровень $n=-2$ (что обеспечивает большую величину матричного элемента перехода). Таким образом, мы связываем линию поглощения $\beta^{*}$ с переходом $-1 \rightarrow-2$ в валентной зоне. Известно, что одним из наиболее интенсивных известных магнитооптических переходов в одиночных КЯ $\mathrm{HgTe} / \mathrm{CdHgTe} \mathrm{является} \mathrm{переход}-2 \rightarrow-1$, обычно обозначаемый символом $\beta$ (см., например, [14,22-25]). В образце с двойной КЯ 150217 мы обнаружили пару линий $\beta_{1}$ и $\beta_{2}$, что связано с „расщеплением“ уровня Ландау $n=-1$ в зоне проводимости вследствие туннельной прозрачности разделительного барьера для электронных состояний. Аналогичная пара линий $\beta_{1}$ и $\beta_{2}$ наблюдается и в образце 150218 (рис. 5,6), расстояние между ними в сильных магнитных полях $(\sim 18$ мэВ при $B=10$ Тл) использовалось в качестве одного из значений для уточнения структурных параметров образца. В темновых условиях высокочастотная линия $\beta_{2}$ практически не видна, а при подсветке она в $\sim 2$ раза уступает линии $\beta_{1}$ по интенсивности. Все это указывает, как и в предыдущем образце, на небольшое расщепление уровня Ландау $n=-2$ из-за различия в ширине КЯ. Отметим также, что линия $\beta_{2}$ несколько шире, чем линия $\beta_{1}$, что можно связать с флуктуациями расстояния между подзонами $E_{2}$ и $H H_{1}$ вследствие неоднородности толщин КЯ по площади структуры.

В отличие от широкозонного образца 150217, в бесщелевом образце 150218 наблюдается еще одна интенсивная линия магнитопоглощения $\gamma^{*}$ (рис. 5,6). Как показывают результаты численных расчетов, из возможных переходов, попадающих в соответствующий интервал энергий, наибольшие матричные элементы имеют переходы $-1 \rightarrow 0$ с группы антипересекающихся уровней $n=-1$ валентной зоны на уровень Ландау $n=0$, происходящий из подзоны $E_{1}$ (см. рис. 4). Таким образом, возникновение этой линии поглощения прямо связано с инверсией зон $E_{1}$ и $H_{1}$. Другим проявлением инверсии зон является возникновение достаточно интенсивной линии поглощения $X$, наблюдаемой в магнитных полях до 1.5 Тл, где она заходит в область остаточных лучей подложки GaAs. Из рис. 6 видно, что эта линия не является „низкочастотным“ продолжением линий $\beta_{1}$ и $\gamma^{*}$. Поскольку при $B \rightarrow 0$ спектральное положение линии $X$ приближается к 20 мэВ, то, учитывая высокое значение фактора заполнения уровней Ландау дырок $v>6$ при $B<1$ Тл, можно заключить, что она связана с переходами между уровнями Ландау из подзон $E_{1}$ и $\mathrm{HH}_{2}$. Как видно из рис. 4, в этой области энергий имеется „Подходящий“ переход $-1 \rightarrow-2$. Однако, как уже отмечалось, в столь небольших магнитных полях должно иметь место сильное возмущение закона дисперсии (и соответственно поведения уровней Ландау), так что в настоящее время (до проведения детального исследования эффектов замешивания состояний на интерфейсе) не представляется возможным дать однозначную интерпретацию наблюдаемой линии магнитопоглощения $X$. Как и для образца 150217 , отметим, что расчеты энергий переходов лишь качественно согласуются с положениями наблюдаемых линий (рис. 6), что также связывается нами с „интерфейсными“ эффектами. Отметим еще, что в образце 150218 заметным является эффект изменения положения многих наблюдаемых линий магнитопоглощения в зависимости от концентрации носителей, изменяемой при подсветке синим светодиодом. Скорее всего, это связано с перестройкой спектра уровней Ландау при изменении профиля квантовой ямы за счет электрического поля, возникающего (или изменяющегося) при пространственном переносе электронов с удаленных центров (ловушек) в квантовую яму, что открывает возможность управления спектром состояний и в принципе фазовым топологическим переходом путем приложения внешнего электрического поля.

Работа выполнена при поддержке гранта 16-12-10317 Российского научного фонда. Авторы признательны Е.Л. Ивченко за обсуждения эффекта замешивания дырочных состояний на гетероинтерфейсах.

\section{Список литературы}

[1] M. Konig, S. Wiedmann, C. Brune, A. Roth, H. Buhmann, L.W. Molenkamp, X.-L. Qi, S.-C. Zhang. Science, 318, 766 (2007).

[2] Y.S. Gui, C.R. Becker, N. Dai, J. Liu, Z.J. Qiu, E.G. Novik, M. Schäfer, X.Z. Shu, J.H. Chu, H. Buhmann, L.W. Molenkamp. Phys. Rev. B, 70, 115328 (2004)

[3] К.Е. Спирин, А.В. Иконников, А.А. Ластовкин, В.И. Гавриленко, С.А. Дворецкий, Н.Н. Михайлов. Письма ЖЭТФ, 92, 65 (2010).

[4] З.Д. Квон, Е.Б. Ольшанецкий, Д.А. Козлов, Н.Н. Михайлов, С.А. Дворецкий. Письма ЖЭТФ, 87, 588 (2008).

[5] G.M. Gusev, E.B. Olshanetsky, Z.D. Kvon, N.N. Mikhailov, S.A. Dvoretsky, J.C. Portal. Phys. Rev. Lett., 104, 166401 (2010)

[6] C. Brüne, C.X. Liu, E.G. Novik, E.M. Hankiewicz, H. Buhmann, Y.L. Chen, X.L. Qi, Z.X. Shen, S.C. Zhang, L.W. Molenkamp. Phys. Rev. Lett., 106, 126803 (2011). 
[7] B.A. Bernevig, T.L. Hughes, S.-C. Zhang. Science, 314, 1757 (2006).

[8] T. Ohta, A. Bostwick, T. Seyller, K. Horn, E. Rotenberg. Science, 313, 951 (2006).

[9] P. Michetti, J.C. Budich, E.G. Novik, P. Recher. Phys. Rev. B, 85, 125309 (2012).

[10] P. Michetti, B. Trauzettel. Appl. Phys. Lett., 102, 063503 (2013).

[11] J.C. Budich, B. Trauzettel, P. Michetti. Phys. Rev. Lett., 112, 146405 (2014).

[12] S.S. Krishtopenko. Тр. ХIX Межсдунар. симп. „Нанофизика и наноэлектроника“, Нижний Новгород, 2015 (Нижний Новгород, Изд-во Нижегородского гос. ун-та им. Н.И. Лобачевского, 2015) т. 2, с. 545.

[13] S.S. Krishtopenko, W. Knap, F. Teppe. arXiv:1604.02423v2

[14] A.V. Ikonnikov, M.S. Zholudev, K.E. Spirin, A.A. Lastovkin, K.V. Maremyanin, V.Ya. Aleshkin, V.I. Gavrilenko, O. Drachenko, M. Helm, J. Wosnitza, M. Goiran, N.N. Mikhailov, S.A. Dvoretskii, F. Teppe, N. Diakonova, C. Consejo, B. Chenaud, W. Knap. Semicond. Sci. Technol., 26, 125011 (2011).

[15] E.G. Novik, A. Pfeuffer-Jeschke, T. Jungwirth, V. Latussek, C.R. Becker, G. Landwehr, H. Buhmann, L.W. Molenkamp. Phys. Rev. B, 72, 035321 (2005).

[16] N.N. Mikhailov, R.N. Smirnov, S.A. Dvoretsky, Y.G. Sidorov, V.A. Shvets, E.V. Spesivtsev, S.V. Rykhlitski. Int. J. Nanotechnol., 3, 120 (2006).

[17] S. Dvoretsky, N. Mikhailov, Y. Sidorov, V. Shvets, S. Danilov, B. Wittman, S. Ganichev. J. Electron. Mater., 39, 918 (2010).

[18] В.Я. Алешкин, Д.Б. Векслер, В.И. Гавриленко, И.В. Ерофеева, А.В. Иконников, Д.В .Козлов, О.А. Кузнецов. ФТТ, 46, 131 (2004).

[19] O. Drachenko, D.V. Kozlov, V.Ya. Aleshkin, V.I. Gavrilenko, K.V. Maremyanin, A.V. Ikonnikov, B.N. Zvonkov, M. Goiran, J. Leotin, G. Fasching, S. Winnerl, H. Schneider, J. Wosnitza, M. Helm. Phys. Rev. B, 79, 073301 (2009).

[20] В.И. Гавриленко, М.С. Жолудев, А.В. Иконников, К.Е. Спирин, Л.С. Бовкун, M. Orlita, F. Teppe, W. Knap, С.А. Дворецкий, Н.Н. Михайлов. Тез. докл. ХІІ Росс. конф. по физике полупроводников, Ершово, 2015 (М., Изд-во ФИАН, ISBN 978-5-902622-31-4), с. 88.

[21] S.A. Tarasenko, M.V. Durnev, M.O. Nestoklon, E.L. Ivchenko, Jun-Wei Luo, Alex Zunger. Phys. Rev. B, 91, 081302(R) (2015).

[22] M. Zholudev, F. Teppe, M. Orlita, C. Consejo, J. Torres, N. Dyakonova, M. Czapkiewicz, J. Wróbel, G. Grabecki, N. Mikhailov, S. Dvoretskii, A. Ikonnikov, K. Spirin, V. Aleshkin, V. Gavrilenko, W. Knap. Phys. Rev. B, 86, 205420 (2012).

[23] M.S. Zholudev, A.V. Ikonnikov, F. Teppe, M. Orlita, K.V. Maremyanin, K.E. Spirin, V.I. Gavrilenko, W. Knap, S.A. Dvoretskiy, N.N. Mihailov. Nanoscale Res. Lett., 7, 534 (2012).

[24] В.В. Платонов, Ю.Б. Кудасов, И.В. Макаров, Д.А. Маслов, О.М. Сурдин, М.С. Жолудев, А.В. Иконников, В.И. Гавриленко, Н.Н. Михайлов, С.А. Дворецкий. ФТП, 49, 1660 (2015).

[25] M. Orlita, K. Masztalerz, C. Faugeras, M. Potemski, E.G. Novik, C. Brune, H. Buhmann, L.W. Molenkamp. Phys. Rev. B, 83, 115307 (2011).

\section{Magnetospectroscopy of double $\mathrm{HgTe} / \mathrm{CdHgTe}$ quantum wells}

L.S. Bovkun ${ }^{+}$, S.S. Krishtopenko ${ }^{+\ddagger}$, A.V. Ikonnikov ${ }^{+\dagger}$, V.Ya. Aleshkin ${ }^{+\dagger}$, A.M. Kadykov ${ }^{+\ddagger}$, S. Ruffenach ${ }^{\ddagger}$, C. Consejo $o^{\ddagger}$, F. Teppe ${ }^{\ddagger}$, W. Knap ${ }^{\ddagger}$, M. Orlita ${ }^{\S}$, B. Piot ${ }^{\S}$, M. Potemski ${ }^{\S}$, N.N. Mikhailov ${ }^{* \dagger}$, S.A. Dvoretskii*, V.I. Gavrilenko ${ }^{+\dagger}$

+ Institute for Physics of Microstructures, Russian Academy of Sciences, 603950 Nizhny Novgorod, Russia

$¥$ Laboratoire Charles Coulomb (L2C), UMR CNRS 5221 \& UM, 34095 Montpellier, France

$\dagger$ Lobachevsky State University of Nizhny Novgorod, 603950 Nizhny Novgorod, Russia

$\S$ Laboratoire National des Champs Magnetiques Intenses (LNCMI-G), CNRS-UJF-UPS-INSA, FR-38042 Grenoble, France

* Rzhanov Institute of Semiconductor Physics, Siberian Branch of Russian Academy of Sciences, 630090 Novosibirsk, Russia

$\dagger$ Novosibirsk State University, 630090 Novosibirsk, Russia

Abstract Magnetoabsorption spectra in double $\mathrm{HgTe} / \mathrm{CdHgTe}$ quantum wells (QWs) with normal and inverted band structure have been investigated. Landau levels in symmetric quantum wells with rectangular potential profile have calculated within Kane 8.8 model. The presence of tunnel transparent barrier is shown to results in state splittings and „doubling“ the main magnetoabsorption lines. At the well width close to the critical one in the single quantum wells structure the band inversion and the gapless band structure arising (as in bilayer graphene) have been demonstrated. The magnetoabsorption line shifts at the carrier concentration change due to the persistent photoconductivity effect have been discovered that is related with potential profile change because of traps charge exchange. The latter opens a possibility to control topological phase transitions in such structures. 